\title{
An investigation of IL-8 degradation in response to PA401 compared to hypertonic saline in bronchoalveolar lavage fluid of Cystic Fibrosis patients
}

\author{
H Kerr ${ }^{*}$, O'Reilly$^{1}$, OJ McElvaney ${ }^{2}$, DA Bergin ${ }^{1}$ \\ From International Conference for Healthcare and Medical Students (ICHAMS) 2013 \\ Dublin, Ireland. 11-12 October 2013
}

\section{Background}

The lung pathogenesis of cystic fibrosis (CF) involves inflammation, airway obstruction and an increased incidence of pulmonary infections. Increased levels of proinflammatory cytokines and chemokines such as interleukin-8 (IL-8) play a pivotal role in sustaining the cycle of inflammation in the CF lung. Glycosaminoglycans (GAGs) possess the ability to bind IL-8 providing protection from proteolytic degradation and maintaining it in an active state leading to sustained neutrophil chemotaxis. It has been shown that hypertonic saline (HTS) disrupts GAG:IL-8 complexes, thus rendering IL-8 susceptible to proteolysis thereby reducing neutrophil chemotaxis. The recombinant IL-8 decoy (PA401) binds glycans with higher affinity $(x$ 40) than native IL-8. In this study, we compared the ability of PA401 and HTS to disrupt IL-8:GAG complexes in CF BALF.

\section{Methods}

IL-8 concentration in CF BALF was determined following exposure to PA401 or HTS by ELISA. PA401 degradation in CF BALF ( \pm protease inhibitors) was examined using gradient SDS-PAGE and Western Blot analysis employing a primary antibody specific for the PA401 decoy (MAB8A12).

\section{Results}

Individual CF BALF samples $(\mathrm{n}=7)$ displayed a high level of variability with regard to IL-8 concentration and response to PA401 or HTS. Exposure of pooled CF BAL to increasing concentrations of PA401 lead to a significant decrease in the level of detectable IL- $8(\mathrm{p}<0.05)$ and

\footnotetext{
${ }^{1}$ Royal College of Surgeons in Ireland, 123 St. Stephen's Green, Dublin, Ireland

Full list of author information is available at the end of the article
}

neutrophil chemotactic efficiency $(30 \%, \mathrm{p}<0.05)$. Significantly reduced levels of IL-8 $(\mathrm{p}<0.05)$ were detected following incubation with PA401 for $4 \mathrm{hr}$ in 6/7 individuals with CF when compared to a PBS control. The level of IL-8 present in BALF following incubation with PA401 was significantly reduced compared to HTS $(\mathrm{p}<0.05)$ in 2/3 CF patients. Western Blot analysis indicated that serine proteases (inhibited by alpha-1 antitrypsin, PMSF and pefabloc) play a major role in degrading PA401.

\section{Conclusions}

The reduced levels of IL-8 in BALF samples treated with PA401 revealed that PA401 is likely to be effective in disrupting IL-8:GAG complexes in the CF lung rendering IL-8 susceptible to proteolysis and thus may be seen as a therapeutic target in the treatment of CF. Further benefits of PA401 are evident as the decoy did not accumulate in CF samples and post IL-8 clearance, it too was degraded by serine and metalloproteases. Clinical application of an IL-8 decoy may serve to decrease the inflammatory burden in the CF lung in vivo.

\section{Authors' details \\ 'Royal College of Surgeons in Ireland, 123 St. Stephen's Green, Dublin, Ireland. ${ }^{2}$ Department of Medicine, Beaumont Hospital, Dublin, Ireland.}

Published: 14 January 2015
doi:10.1186/1753-6561-9-S1-A12

Cite this article as: Kerr et al: An investigation of IL-8 degradation in response to PA401 compared to hypertonic saline in bronchoalveolar lavage fluid of Cystic Fibrosis patients. BMC Proceedings 2015 9(Suppl 1): A12. 